\title{
Feasibility of teledermatology in the eradication of leishmaniasis from Yemen project
}

\author{
Mohamed Ahmed Al-Kamel \\ Founding chair, Regional Leishmaniasis Control Center (RLCC), Yemen
}

\begin{abstract}
This review demonstrates the feasibility of "Teledermatology" in delivering skin care services to patients with leishmaniasis disease in underserved areas, utilizing simple technology applications. "Teledermatology program" of the Eradication of Leishmaniasis from Yemen Project (ELYP) has been discussed, as an example.
\end{abstract}

\begin{abstract}
Abbreviations: ELYP: Eradication of Leishmaniasis from Yemen Project; RLCC: Regional Leishmaniasis Control Center; ISD: International Society of Dermatology; AAD: American Academy of Dermatology; EADV: European Academy of Dermatology and Venereology; ILDS: International League of Dermatologic Societies
\end{abstract}

\section{Introduction}

Telemedicine, a branch of e-health, is one of wide opportunities offered by the advance in both the medical sciences and electronic communication. It is not a specific technology, but a dynamic process that utilizes interactive telecommunications technology to share digitized medical data between two parties to provide patient care, as well as health education for patients and health care providers at a distance.

Teledermatology, that can be defined as the remote delivery of skin care services and information using telecommunications technology, is indeed unique and most frequently used service of telemedicine due to the visual nature of skin and skin diseases. Generally, teledermatology can be categorized, upon the utilized technology, platform and application used, into three main types, (1) live-interactive (synchronous) teledermatology; (2) store-forward (asynchronous) teledermatology; and (3) Hybrid (combined) teledermatology [1].

Live-interactive teledermatology usually utilizes web-based communications and requires the presence of both parties at the same time; it usually employ telephone call and videoconferencing technology for direct real time interaction. Store-forward teledermatology is the most commonly employed method due to its asynchronous nature; it does not require the presence of both parties at the same time; and hence, clinical data and skin photographs are captured, stored and forwarded at a convenient time for later review. Hybrid teledermatology covers features of both the above-mentioned types; it performs instant live-interactive as well as sore and forward teledermatology operations.

\section{Adoption of Teledermatology in Eradication of Leishmaniasis from Yemen Project (ELYP)}

To implement strategies of the ISD- and AAD- supported, community-led, Eradication of Leishmaniasis from Yemen Project (ELYP) to combat the neglected endemic leishmaniasis disease that predominantly prevail among poor rural children and women, Regional Leishmaniasis Control Center (RLCC) was founded during April 2013 as a first phase of the project ELYP. Immediately, as the second phase of the project ELYP, RLCC released several integrated programs for the same purpose. Because of the complex geography of Yemen, weak infrastructures, scarce medical facilities in rural areas, deteriorating economic status, high cost of health care, conflicts and instability, Teledermatology program was adopted as a standalone innovative program that may provide a communication tool between caregiver each other at different regional centers/units, them and their instructors and project supporters abroad and between leishmaniasis patients in distant disadvantaged areas and their treating doctors at center's regional clinics [2-4].

\section{RLCC's teledermatology program resources}

Due to scarcity in resources, RLCC has not relied on specific telemedicine-specific hardware or software to implement teledermatology program, but it utilized all available resources as to support the program.

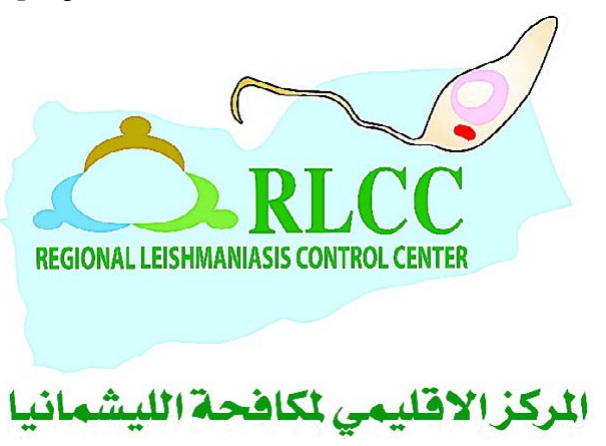

Figure 1. Regional Leishmaniasis Control Center logo.

Correspondence to: Dr. Mohamed Ahmed Al-Kamel, Regional Leishmaniasis Control Center (RLCC), P.O.Box 12692, Sana'a,Yemen, E-mail: dralkamel@ hotmail.com

Key words: teledermatology, telemedicine, leishmaniasis, ELYP, RLCC, Yemen

Received: January 29, 2017; Accepted: February 25, 2017; Published: February 28,2017 


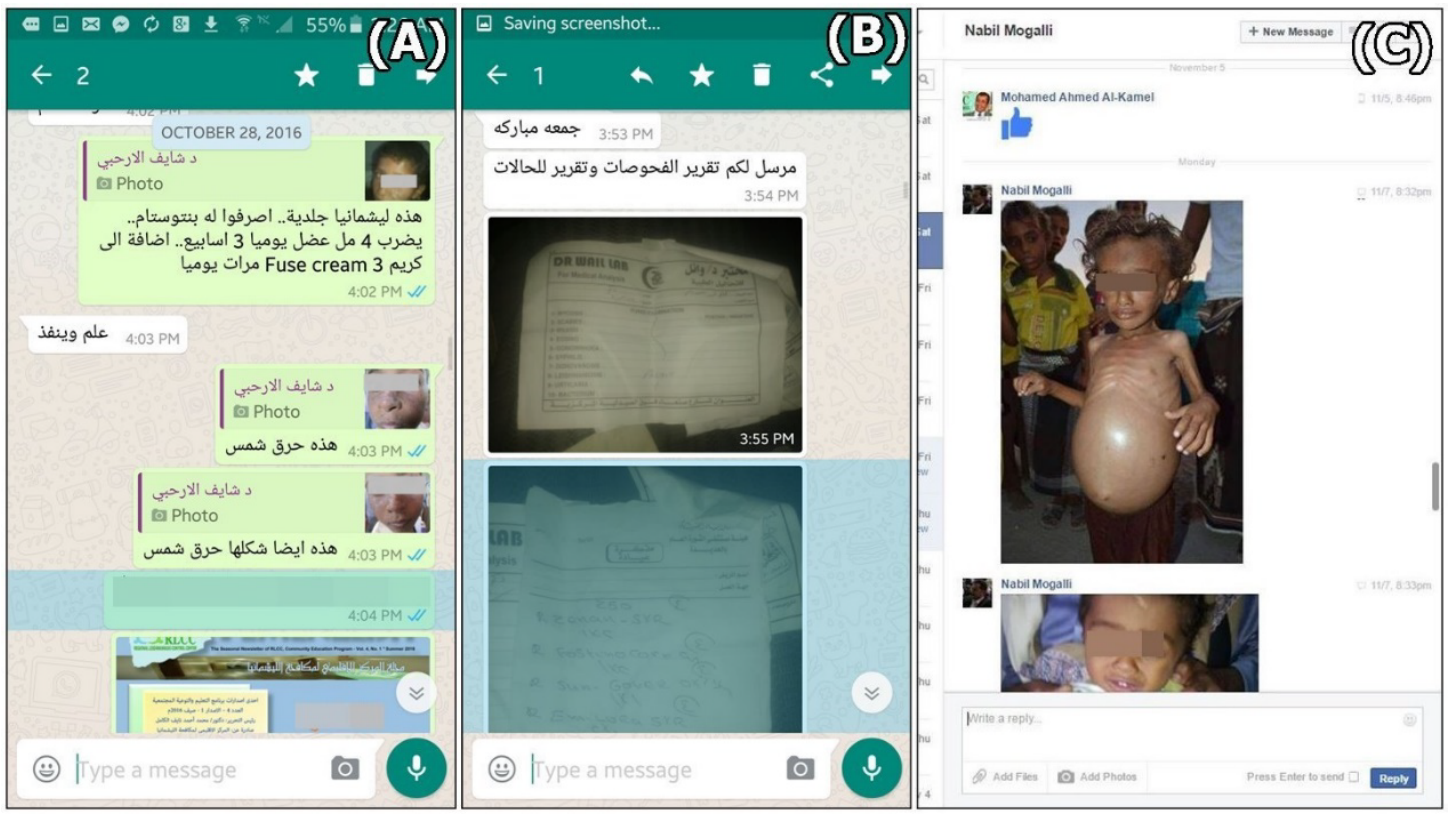

Figure 2.Utilizing Social Media (WhatsApp "A\&B" and Facebook Messenger "C") for Teledermatology by Regional Leishmaniasis Control Center (RLCC) to control leishmaniasis disease in Yemen. Images and information exchange among RLCC's staff.

Although desktop computer, telephone, mobile devices (eg., laptop; tablet; cellular; and smartphone) and mobile device-based services (eg., "SMS"; phone calling; internet-based software applications and social media) were adopted in the program, smartphones are the most commonly used devices and "WhatsApp" is the most commonly used channel by all parties, followed by "phone calling" which is commonly used by distant poor patients, "Facebook" and "Email" which are most commonly used by RLCC staff and colleagues.

Despite mobile teledermatology has been the strategy widely used by RLCC, a bundle of technical and economic issues make synchronous sharing (live-interactive teledermatology) an unreliable option and "store-forward teledermatology" the dominant type. Internet-based applications offering store-forward service introduced a solution to remotely send text, images, voice and even videos for consultation discussion and treatment planning among RLCC's patients and physicians or among physicians each other.

Likewise, RLCC utilizes Teledermatology program to regularly send educational materials and promote its free services (eg., medicine donation) to all local mobile networks subscribers in Yemen (eg., Yemen Mobile) via the bulk "SMS" service, as well as via mass media and internet-based social media.

\section{RLCC's Teledermatology program challenges}

Teledermatology program of RLCC has been facing spectral obstacles in providing its ambitious services in Yemen. Scarce financial resources; economic, cultural and technical limitation (eg., low-speed ADSL and 3G networks); shortage of electricity; lack of computing mobile devices and mobile networks coverage in rural areas; patient's unfamiliarity; and illiteracy are Some of the challenges and barriers.

\section{RLCC's teledermatology program impact}

RLCC's Teledermatology program does remotely, (1) provide patients with referral services, early diagnosis and intervention advice, treatment follow-up, prognosis assessment, prevention recommendation and awareness-raising education; (2) provide center's staff with patient data from remote areas and facilitate inter-physician coordination; (3) provide the project with a communication channels with its partners and supporters abroad.

Moreover, Teledermatology program is effectively used to attract scientific global attention to unique issues linked to leishmaniasis disease in Yemen (such as, endemicity; mortality; morbidity; scarcity of medicine; and issues related to children and women as a group at higher risk), to enrich the global epidemiological profile of leishmaniasis and as a mean of attracting medicine and medical support for those needy patients. As a result, many studies of leishmaniasis disease in Yemen have been conducted, globally published, and the project ELYP has attracted numerous technical and scientific support, research awards and anti-leishmania medicament donations from some interesting international agencies, such as ISD, AAD, ILDS and Stiefel/GSK 4 . Teledermatology program has supported other programs of RLCC, such as Community Education program, Research program, Surveillance program and Medicine-For-All program, and thus played a significant role in the ELYP's strategies to combat leishmaniasis and its related consequences, including leishmaniasis-related stigmata.

\section{In conclusion}

The developing Teledermatology program of RLCC, using simple available technology resources, has played a key role in offering patients with leishmaniasis with free access to what was unavailable and costly unaffordable treatment and quick access to consultation and support services. It has improved lives and saved unnecessary transportation cost of patients with leishmaniasis at rural disadvantaged areas, improved patients' documentation, raised remote education and community awareness of the disease, enhanced staff knowledge and enhanced research capabilities of leishmaniasis in Yemen.

\section{References}

1. Pathipati A, Armstrong A (2011) Teledermatology: Outcomes and Economic Considerations, Telemedicine Techniques and Applications. InTech Open, Coratia. 
2. Al-Kamel MA (2016) Impact of leishmaniasis in women: a practical review with an update on my ISD-supported initiative to combat leishmaniasis in Yemen (ELYP). Int J Womens Dermatol 2: 93-101.

3. Al-Kamel MA (2013) Regional Leishmaniasis Control Center (RLCC) Yemen. 22 $2^{\text {nd }}$
European Academy of Dermatology and Venereology Congress, 2013, Istanbul, Turkey.

4. Al-Kamel MA (2017) Stigmata in cutaneous leishmaniasis: Historical and new evidence-based concepts. Our Dermatol Online 8: 81-90.

Copyright: (C2017 Al-Kamel MA. This is an open-access article distributed under the terms of the Creative Commons Attribution License, which permits unrestricted use, distribution, and reproduction in any medium, provided the original author and source are credited. 